\title{
A saddle-node bifurcation model of magnetic reconnection onset
}

\author{
P. A. Cassak \\ M. A. Shay \\ J. F. Drake
}

Follow this and additional works at: https://researchrepository.wvu.edu/faculty_publications

\section{Digital Commons Citation}

Cassak, P. A.; Shay, M. A.; and Drake, J. F., "A saddle-node bifurcation model of magnetic reconnection onset" (2010). Faculty Scholarship. 39.

https://researchrepository.wvu.edu/faculty_publications/39 


\title{
A saddle-node bifurcation model of magnetic reconnection onset
}

\author{
P. A. Cassak, ${ }^{1}$ M. A. Shay, ${ }^{2}$ and J. F. Drake ${ }^{3}$ \\ ${ }_{1}^{1}$ Department of Physics, West Virginia University, Morgantown, West Virginia 26506, USA \\ ${ }^{2}$ Department of Physics and Astronomy, University of Delaware, Newark, Delaware 19716, USA \\ ${ }^{3}$ IREAP, University of Maryland, College Park, Maryland 20742, USA
}

(Received 12 February 2010; accepted 5 May 2010; published online 11 June 2010)

\begin{abstract}
It was recently shown that magnetic reconnection exhibits bistability, where the Sweet-Parker (collisional) and Hall (collisionless) reconnection solutions are both attainable for the same set of system parameters. Here, a dynamical model based on saddle-node bifurcations is presented which reproduces the slow to fast transition. It is argued that the properties of the dynamical model are a result of the Hall effect and the dispersive physics associated with it. Evidence from resistive two-fluid and Hall magnetohydrodynamics simulations are presented that show that the time evolution agrees with the dynamical model, the outflow speed is correlated with the dispersive physics due to the Hall effect, and bistability persists in the absence of electron inertia. (C) 2010 American Institute of Physics. [doi:10.1063/1.3435269]
\end{abstract}

\section{INTRODUCTION}

The nonlinear dynamics of magnetic reconnection, the process which is thought to enable rapid magnetic energy release in solar eruptions and fusion devices, has long been a topic of interest. ${ }^{1,2}$ Recently, it was shown that reconnection is bistable for a wide range of Lundquist numbers, and that bistability leads to hysteresis-like behavior. ${ }^{3,4}$ The two stable states are the (collisional) Sweet-Parker solution ${ }^{5,6}$ which is very slow and the (collisionless) Hall solution ${ }^{7-9}$ which is fast. A third solution, which is unstable to small perturbations and lies "between" the two stable solutions, was predicted and identified numerically. ${ }^{10} \mathrm{~A}$ consequence of bistability is that the transition from the collisional to collisionless states is catastrophic, which prompted the suggestion that this is the underlying physics of abrupt onset seen in observations. ${ }^{3,11-13}$

A number of recent studies addressed the nonlinear dynamics of reconnection using Sweet-Parker-type scaling analyses. Flux pileup and time dependence were incorporated into the Sweet-Parker theory. ${ }^{14}$ A similar approach was employed for reconnection within electron magnetohydrodynamics (EMHD) (without electron inertia), ${ }^{15}$ pair plasmas, ${ }^{16}$ Hall-MHD (without electron inertia), ${ }^{17-21}$ two-fluid (HallMHD with electron inertia), ${ }^{22}$ and EMHD with electron inertia. $^{23}$ These analyses employed a resistivity or hyperresistivity (electron viscosity) to break the frozen-in condition, though two recent studies used off-diagonal pressure tensor terms in scaling analyses within the two-fluid ${ }^{24}$ and pair plasma ${ }^{25}$ models. A common result is that resistive reconnection has elongated layers, but hyper-resistive reconnection has either elongated (slow) or $x$-type (fast) layers depending on macroscopic driving. (Note that many authors use "fast" to mean "weakly dependent on dissipation mechanism," whereas we use the more restrictive definition of "weakly dependent on dissipation mechanism and system size.") Bistability was identified in some of these results. Since bistability occurred in the scaling analyses when electron inertia was present but not otherwise, ${ }^{17,19,23}$ it was suggested $^{23}$ that electron inertia causes bistability. Previous simulations ${ }^{3,4}$ were ambiguous on this point because both the Hall effect and electron inertia were employed simultaneously. This presents an unresolved question about the physical cause of bistability and hysteresis.

In this paper, we present a physical model showing that bistability and hysteresis are caused by the Hall effect. First, we motivate that the dynamics can be described as a saddlenode bifurcation ${ }^{26}$ which brings steady-state solutions into and out of existence as a control parameter varies. Then, we argue that the saddle-node bifurcation is a result of the dispersive effects introduced by the Hall term and present a physical explanation of why this is the case. Finally, we present evidence from two-fluid and Hall-MHD numerical simulations that such a model is borne out numerically. In particular, we show that the time evolution of the current sheet displays the properties of a saddle-node bifurcation. We show that the outflow speed during the transition is closely coupled to the dispersive character of the phase speed of waves in Hall-MHD. ${ }^{7,27}$ We then use Hall-MHD simulations without electron inertia to demonstrate that the Hall effect controls the observed dynamics rather than electron inertia. We conclude with a discussion of implications of the theory and discuss how inclusion of secondary islands in SweetParker reconnection affects the results.

\section{THEORY}

A summary of reconnection dynamics as learned in Refs. 3 and 10 is sketched in Fig. 1(a). The normalized reconnection rate $E^{\prime}=c E / c_{A} B_{x}$ is plotted as a function of collisionality parameter $\eta^{\prime}=\eta c^{2} / 4 \pi c_{A} d_{i}$, where $E$ is the reconnection electric field, $B_{x}$ is the reconnecting field immediately upstream of the current layer, $\eta$ is the (Spitzer) resistivity, $c_{A}$ is the Alfvén speed based on $B_{x}$, and $d_{i}$ is the ion inertial length. We define $x$ as the outflow direction, $y$ as the inflow direction, and $z$ as the out-of-plane direction. At high $\eta^{\prime}$, only the Sweet-Parker solution exists. At low $\eta^{\prime}$, only the 


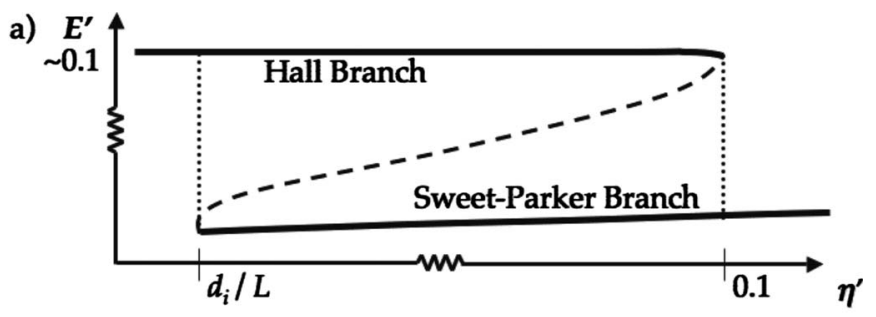

b)

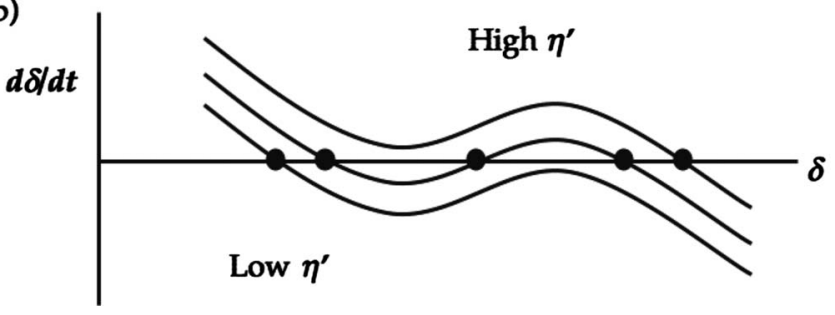

FIG. 1. (a) Schematic summary of the nonlinear dynamics of magnetic reconnection found in Refs. 3 and 10. The dashed line shows a predicted unstable steady-state reconnection solution. (b) Bifurcation model of reconnection which reproduces the behavior in panel (a). Shown is a schematic phase portrait for $\delta$. As control parameter $\eta^{\prime}$ decreases, the curve moves down. Heavy dots denote steady-state solutions.

Hall solution exists. At intermediate $\eta^{\prime}$, both exist and the system is bistable. The dashed line corresponds to the unstable solution found in Ref. 10.

In trying to understand the cause of the observed transition dynamics, we sketch the simplest dynamical model which reproduces the observed behavior. A bifurcation model is plotted schematically in Fig. 1(b) as a phase portrait (a variable plotted against its time derivative) for various values of a control parameter. We identify the dynamical variable as the half-thickness of the current sheet $\delta$ and the control parameter as the collisionality parameter $\eta^{\prime}$. We show later why this choice of variables and parameters was made.

We now motivate that the dynamics described by Fig. 1(b) reproduces the observed dynamics. Steady-state equilibria (fixed points) occur where $d \delta / d t=0$ and are marked by heavy dots. The uppermost curve is for large collisionality $\eta^{\prime}$, with a single (stable) steady-state solution at relatively high $\delta$, corresponding to the Sweet-Parker solution. As $\eta^{\prime}$ decreases, the curve moves down and two fixed points (one stable, one unstable) are borne in a saddle-node bifurcation. ${ }^{26}$ The stable fixed point at small $\delta$ corresponds to the Hall solution. For a range of $\eta^{\prime}$, there are two distinct stable steady-state solutions as shown in the middle curve, i.e., there is bistability. The existence of the unstable solution ${ }^{10}$ between the two stable solutions appears naturally in this model. As $\eta^{\prime}$ continues to decrease, the curve moves lower, and the unstable and Sweet-Parker fixed points approach each other, coalesce, and disappear in a second saddle-node bifurcation. For low collisionality $\eta^{\prime}$, the only fixed point is the Hall solution, as is shown in the lowest curve in Fig. 1(b). Therefore, this simple dynamical picture reproduces the complicated observed dynamics.

While Fig. 1(b) is potentially a valid description, it does not explain why such a description should arise. Before arguing that the key physics is the Hall effect, we review the physics of the standard Sweet-Parker solution. In this solution, convection outside the layer balances diffusion within it. If convection exceeds diffusion, the layer becomes thinner; if diffusion exceeds convection, the layer becomes broader. This leads one to posit an evolution equation for $\delta$ of the form ${ }^{28}$

$$
\frac{d \delta}{d t}=-v_{\mathrm{in}}+\frac{\eta c^{2}}{4 \pi \delta},
$$

where $v_{\text {in }}$ is the inflow speed. Formally, this can be derived from a scaling analysis applied to the out-of-plane $(z)$ component of the resistive MHD Ohm's law

$$
E_{z}+\left(\frac{\mathbf{v} \times \mathbf{B}}{c}\right)_{z}=\eta J_{z} .
$$

Using $J_{z} \sim c B_{x} / 4 \pi \delta$ from Ampère's law, $(\mathbf{v} \times \mathbf{B})_{z} \sim v_{\text {in }} B_{x}$, and $E_{z} \sim-(1 / c) \partial A_{z} / \partial t$ with $A_{z} \sim B_{x} \delta$ and assuming that $B_{x}$ is relatively constant and being careful with minus signs, one arrives at Eq. (1). This result is similar to that derived independently in Ref. 14.

Using continuity, one finds $v_{\text {in }} \sim \delta c_{A} / L_{\mathrm{SP}}$, where $L_{\mathrm{SP}}$ is the length of the Sweet-Parker current layer in the outflow direction. This leaves

$$
\frac{d \delta}{d t}=-\frac{\delta c_{A}}{L_{\mathrm{SP}}}+\frac{\eta c^{2}}{4 \pi \delta} .
$$

As a check, the fixed point (where $d \delta / d t=0$ ) is indeed the standard Sweet-Parker solution, $\delta=\left(\eta c^{2} L_{\mathrm{SP}} / 4 \pi c_{A}\right)^{1 / 2}$. The phase space portrait starts at positive infinity for small $\delta$ and decreases monotonically to negative infinity for large $\delta$. Therefore, there is only one steady solution in resistive MHD reconnection, the Sweet-Parker solution. (In particular, the robust dynamics observed in Refs. 3 and 10 is not captured.) The Sweet-Parker solution is stable to small perturbations in $\delta$. This is because a small decrease in the layer leads to a decrease in convection and an increase in diffusion, so the layer's response is to broaden, which opposes the perturbation.

With this interpretation of Sweet-Parker reconnection in mind, consider the dynamics described in Fig. 1(b). It is known that Hall reconnection is marked by the decoupling of ions from the magnetic field and electrons at a length scale of the thermal or inertial ion gyroradius. ${ }^{29}$ The current sheet thickness is on electron scales, ${ }^{30}$ and while resistive effects may play a small role, the bulk of the reconnection electric field is balanced by portions of the off-diagonal pressure tensor. ${ }^{31}$ The unstable solution exists at a current sheet width that is intermediate between electron and ion length scales. ${ }^{10}$ As a consequence, ions have decoupled from the field, but the electrons have not. In this region, electron inertia effects are negligible, which means that the only possible physical mechanism allowing a balance of the reconnection electric field is for electron convection (ion convection plus the Hall term) to balance resistive diffusion. The mechanism for Ohm's law balance is plotted schematically in Fig. 2 for the (a) Sweet-Parker, (b) unstable, and (c) Hall solutions. Red lines denote oppositely directed magnetic fields, blue lines are ion flow, and black lines are electron flow. The blue box 


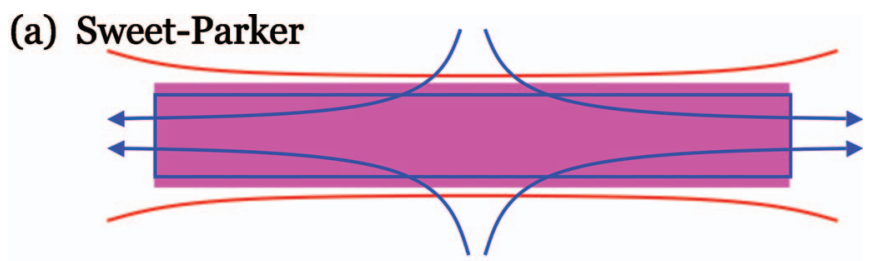

(b) Unstable

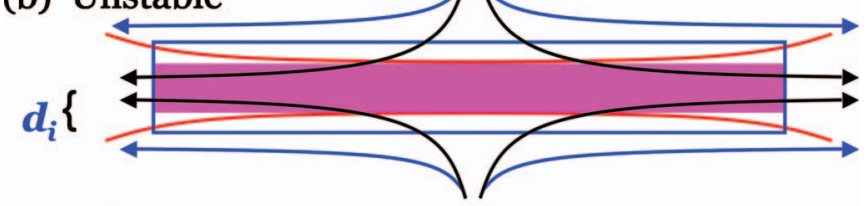

(c) Hall

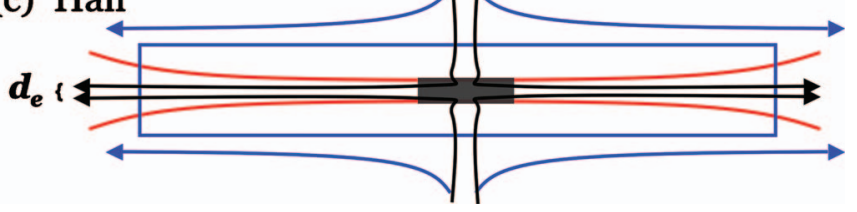

FIG. 2. (Color) Schematic of the governing physics for the (a) SweetParker, (b) unstable, and (c) Hall reconnection solutions. Red lines are oppositely directed magnetic field lines, blue lines denote ion flow, and the black lines denote electron flow. The purple box denotes a resistive diffusion region, while the black box denotes a diffusion region dominated by kinetic effects.

denotes ion gyroscales. The shaded boxes denote the dissipation region (defined as where the frozen-in condition is violated), with purple for resistive diffusion and black for kinetic effects such as pressure tensor terms.

The reconnection rate of the unstable solution can readily be computed in the limit where the ion inflow is small compared to the electron inflow. Balancing the Hall term and the resistive term in the out-of-plane $(z)$ component of the generalized Ohm's law gives $J_{y} B_{x} /$ nec $\sim \eta J_{z}$. Using $J_{y} \sim c B_{z} / 4 \pi L$ and $J_{z} \sim c B_{x} / 4 \pi \delta$ from Ampere's law gives

$$
E^{\prime} \sim \eta^{\prime}
$$

where $E^{\prime}=c E / B_{\text {up }} c_{A_{\text {up }}} \sim \delta / L$ is the normalized reconnection rate, $L$ is the length of the dissipation region in the outflow direction, and we have assumed that $B_{z} \sim B_{x}$. This prediction is in good agreement with the simulations in Ref. 10. Note that $E^{\prime}$ scales like $\eta$ (as opposed to $\eta^{1 / 2}$ for Sweet-Parker), but is independent of the system size $L_{\mathrm{SP}}$, so is actually faster than the Sweet-Parker rate, as is seen in Fig. 1(a). This scaling analysis has been previously carried out, ${ }^{15,17-19,22,23}$ but it has not been appreciated that this solution corresponds to an unstable, and thus physically unrealizable, solution.

The fact that the unstable solution is a balance between electron convection and resistive diffusion has profound implications about reconnection dynamics. As in the SweetParker model, convection tends to reduce the thickness of the layer, while diffusion broadens it. For the unstable solution to be unstable, it must be true that compressing the layer leads to an increase in convection that overcomes the increase in diffusion, so runaway toward smaller length scales occurs. The runaway process stops only when additional physics, such as off-diagonal elements of the pressure tensor, become important at electron scales, which is the Hall solution. (In two-fluid simulations of Hall reconnection, the run- away process is often stopped using an explicit high order dissipation term such as hyperviscosity or through numerical dissipation because off-diagonal pressure tensor terms are absent from the model.) The physical cause of this runaway lies in the Hall effect. Reconnection outflow is driven by the straightening of newly reconnected field lines, which at subion gyroradius scales is the whistler wave. The whistler wave is dispersive, so is faster at smaller scales. Upon squeezing the layer, the outflow speed increases. ${ }^{7,32}$ By continuity, the increase in outflow speed leads to an increase in inflow speed. This increase in convection due to Hall physics is sufficient to overpower diffusion and lead to runaway.

We conclude that the Hall effect is crucial to enabling the bistability and hysteresis. For bistability, in terms of the phase portrait in Fig. 1(b), the Hall effect increases the inflow speed above where it would be in the absence of the Hall effect, which from Eq. (1) makes $d \delta / d t$ less negative between ion and electron length scales. This leads to the "bump" in the phase portrait which gives rise to saddle-node bifurcations as $\eta^{\prime}$ is varied. For hysteresis, the resistive term balances the reconnection electric field during Sweet-Parker reconnection. Once a transition to Hall reconnection is made, the reconnection electric field is orders of magnitude faster, so the resistive term cannot play much of a role. Changing $\eta^{\prime}$ by a small amount after Hall reconnection starts has no effect on the system, which is the physical cause of hysteresis. Therefore, the Hall effect and the dispersive behavior introduced by it are instrumental in leading to the rich dynamics observed in previous simulations.

\section{NUMERICAL SIMULATIONS}

The bifurcation model in terms of saddle-node bifurcations makes predictions that can be directly tested with numerical simulations. In this section, we present three pieces of evidence that support the model in the previous section.

In what follows, magnetic fields, densities, velocities, lengths, electric fields, and resistivities are normalized to $B_{0}, n_{0}$, the Alfvén speed $c_{A 0}=B_{0} /\left(4 \pi m_{i} n_{0}\right)^{1 / 2}$, the ion inertial length $d_{i}=\left(m_{i} c^{2} / 4 \pi n_{0} e^{2}\right)^{1 / 2}, \quad E_{0}=c_{A 0} B_{0} / c$, and $\eta_{0}$ $=4 \pi c_{A 0} d_{i} / c^{2}$.

\section{A. Time evolution of the dissipation region thickness}

Figure 1(b) makes a prediction about the time evolution of the thickness of the dissipation region. We can test this prediction using results from the two-fluid numerical simulations presented in Ref. 3. The simulations are described fully in Ref. 3, but we relay the salient details here. We employ the double Harris sheet configuration with doubly periodic boundary conditions in a system with size of $409.6 \times 204.8 d_{i}$ with grid scale of $0.1 d_{i}$ and initial Harris sheet width of $2 d_{i}$ using the two-fluid code F3D. ${ }^{33}$ The electron mass is $m_{e}=m_{i} / 25$ and the fourth order diffusion coefficient (hyperviscosity) is $2 \times 10^{-5}$. Convergence tests have been performed. In these simulations, Sweet-Parker reconnection was attained with a resistivity of $\eta=0.015$ despite the presence of the Hall term. Then, the resistivity was lowered below the threshold value. This creates a system that is 

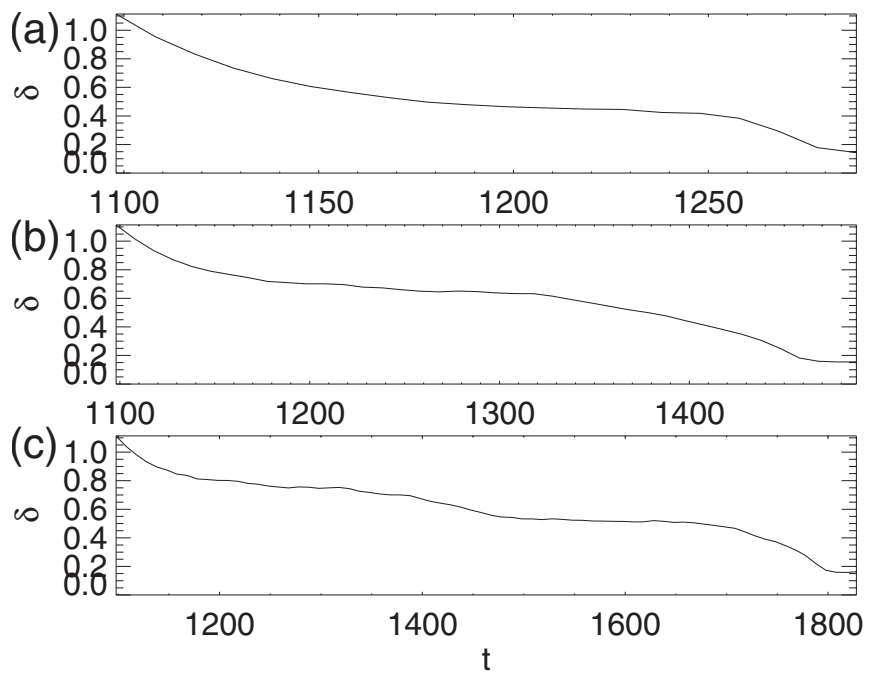

FIG. 3. Simulation data of the dissipation region thickness $\delta$ vs time $t$ for simulations from Ref. 3 in which a transition from Sweet-Parker to Hall reconnection is made when $\eta$ is lowered from 0.015 to (a) 0.003 , (b) 0.007 , and (c) 0.009 .

out of equilibrium which will transition to Hall reconnection, so its time evolution can be studied.

During the transition, the thickness of the layer $\delta$ (defined as the half-width at half-maximum of the out-of-plane current density $J_{z}$ ) is measured as a function of time. The behavior of $\delta$ versus time appears in Fig. 3 for runs with the lowered resistivities $\eta=0.003,0.007$, and 0.009 , seen as a collapse from ion $(\delta \sim 1)$ to electron $\left(\delta \sim d_{e}=0.2\right)$ length scales. The resultant phase portraits of $\delta$ are shown in Fig. 4, with the solid black, blue, and red lines for the $\eta=0.003$, 0.007 , and 0.009 simulations, respectively. Clearly, they qualitatively resemble the small $\eta^{\prime}$ curve in Fig. 1(b), which supports the saddle-node bifurcation picture of the dynamics. For completeness, the dot-dashed line is for a simulation in which a transition from Hall to Sweet-Parker reconnection occurs when the resistivity is suddenly increased to 0.0225 .

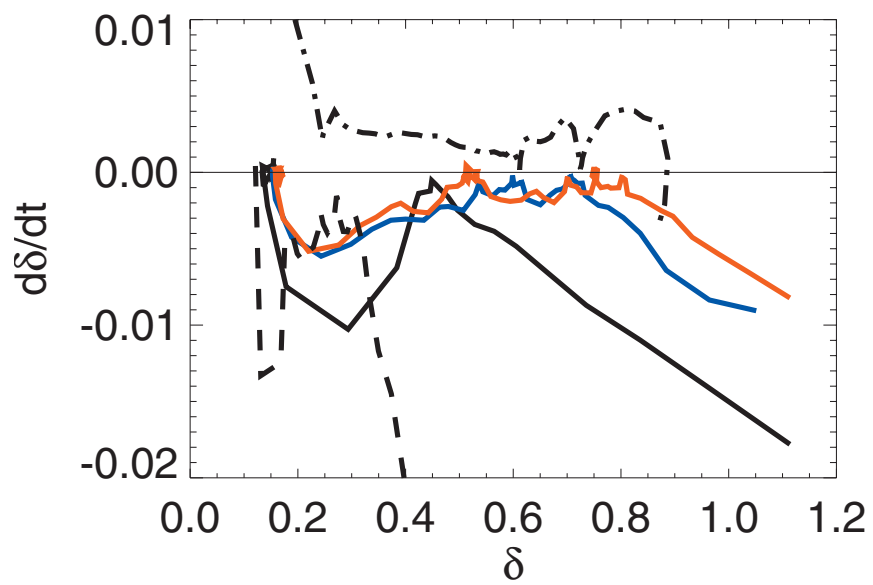

FIG. 4. (Color) Phase portrait for $\delta$ constructed from the data in Fig. 3. The solid lines are for $\eta=0.003$ (black), 0.007 (blue), and 0.009 (red). The results are similar to Fig. 1(a). The dot-dashed line is for a transition from Hall to Sweet-Parker reconnection when the resistivity is increased to 0.0225 . The dashed line is for a run with zero electron mass, showing that the behavior persists.

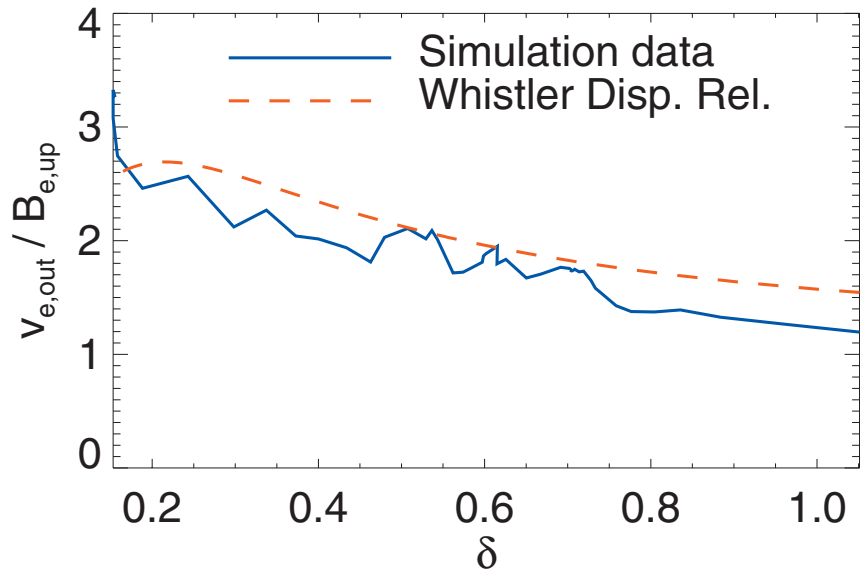

FIG. 5. (Color) Electron outflow speed $v_{e, \text { out }}$ normalized to the magnetic field upstream of the electron layer $B_{e \text {,up }}$ as a function of current layer half-thickness $\delta$ during the transition from Sweet-Parker to Hall reconnection from Ref. 10. The dashed line gives the phase speed $v_{\text {phase }}$ of Hall-MHD waves from Eq. (5) using $k \sim 1 / \delta$.

As expected, $\delta$ increases in time until the system reaches the Sweet-Parker solution. A quantitative comparison with Eq. (3) is not feasible because the upstream magnetic field changes in time, an effect not included in the present analysis. Such effects are considered in Ref. 14.

\section{B. Relation of outflow speed and Hall-MHD wave phase speed}

It was argued in Sec. II that the cause of bistability is the nature of the dispersive behavior of Hall physics. In particular, it was argued that the outflow is driven by the wavelike character of the newly reconnected field lines. Here, we show evidence that the outflow speed during a transition from Sweet-Parker to Hall reconnection is correlated with the phase speed of the whistler wave in two-fluid theory.

The phase speed $v_{\text {phase }}$ of a two-fluid wave with wavenumber $\mathbf{k}$ parallel to the equilibrium field is 33,34

$$
v_{\text {phase }}^{2}=\frac{\omega^{2}}{k^{2}}=\frac{c_{A}^{2}}{D}\left(1+\frac{k^{2} d_{i}^{2}}{2 D}+\sqrt{\frac{k^{2} d_{i}^{2}}{D}+\frac{k^{4} d_{i}^{4}}{4 D^{2}}}\right),
$$

where $D=1+k^{2} d_{e}^{2}$ and $d_{e}$ is the electron inertial scale. This gives Alfvén waves at small $k$, whistler waves for intermediate $k$, and electron cyclotron waves for large $k$.

Results are shown in Fig. 5 for the simulation in which a transition from Sweet-Parker to Hall reconnection occurred when $\eta$ was lowered from 0.015 to 0.007 . During this transition, we measure the electron outflow speed $v_{e, \text { out }}$ (the maximum of electron velocity in the outflow direction in a cut through the $X$ line), $\delta$ (the half-width at half-maximum of the out-of-plane current density $J_{z}$ ), and the magnetic field $B_{e, \text { up }}$ at the electron layer (the magnetic field $\delta$ upstream of the $X$ line).

The solid (blue) line is $v_{e, \text { out }}$ normalized to $B_{e \text {,up }}$ as a function of $\delta$ during the transition. On the same plot, the phase speed of the two-fluid wave from Eq. (5) is plotted as the (red) dashed line, using $k \sim 1 / \delta$. Clearly, the outflow speed is well described by the phase speed of the two-fluid wave throughout the transition from Sweet-Parker to Hall 


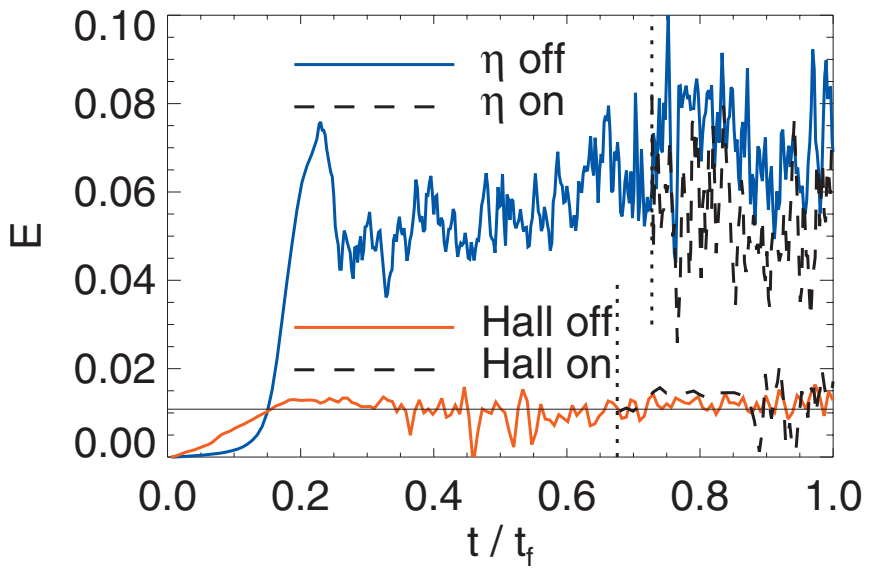

FIG. 6. (Color) Demonstration of bistability in the absence of electron inertia. Reconnection rate $E$ vs time $t$ normalized to the final time $t_{f}$ of the simulation for the runs described in the text. The vertical dashed lines show when the added effects were enabled. The horizontal line gives the predicted Sweet-Parker rate for these parameters.

reconnection, spanning MHD length scales down to electron length scales. Using a more precise value of $k \sim \pi / 2 \delta$ (Ref. 33) merely shifts the theoretical curve but does not change that the two curves follow each other. This provides evidence that the outflow speed increases with thinner layers within ion gyroradius scales due to the Hall effect.

\section{Dependence of bistability on electron inertia}

To determine whether bistability occurs as a result of the Hall effect or electron inertia, we perform new simulations similar to those in Ref. 3 using the massively parallel code F3D ${ }^{33}$ but without electron inertia. Instead of electron inertia, we use a fourth order hyper-resistivity with coefficient $D_{4}=8 \times 10^{-5}$ in all of the equations. This value of $D_{4}$ is four times larger than in Ref. 3. This term regularizes the whistler mode at small scales in the absence of electron inertia. Even with the larger $D_{4}$, a time step 20 times smaller than in Ref. 3 is required to ensure stability.

The two-dimensional periodic domain has size $L_{x} \times L_{y}$ $=409.6 \times 204.8$ with grid scale of 0.1 . The initial equilibrium is two Harris sheets in a double tearing mode configuration with initial current sheet thickness of 1.2. Initially, ions are stationary and pressure is balanced with a nonuniform density $n$ asymptoting to 1 far from the sheet. There is no initial out-of-plane (guide) magnetic field. The plasma is isothermal with constant and uniform temperature $T=1$. There is no viscosity. Initial random perturbations on the magnetic field of amplitude $5 \times 10^{-6}$ break symmetry so secondary magnetic islands are ejected. The resistivity $\eta=0.012$ is constant and uniform when used. This value of $\eta$ ensures that we are in the bistable range, but also outside of the range of continual secondary island formation. We address the formation of secondary islands in Sec. IV. Reconnection is initiated using a coherent field perturbation with amplitude of $4 \times 10^{-3}$.

A simulation is begun with nonzero $\eta$ but the Hall term turned off. The reconnection rate $E$, measured as the time rate of change in magnetic flux between the $X$ and $O$ line, is

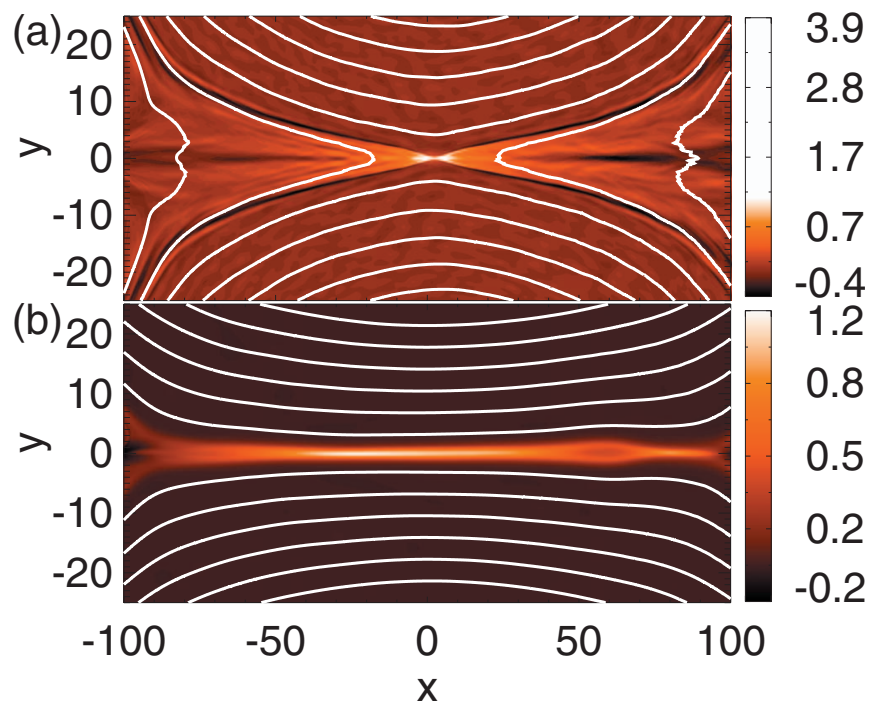

FIG. 7. (Color) Out-of-plane current density $J_{z}$ for the (a) fast and (b) slow stable solutions in Fig. 6 in the absence of electron inertia. For clarity, only a portion of the computational domain is plotted with an altered aspect ratio and the color table in (a) has been stretched.

plotted as the (red) solid line in Fig. 6 as a function of time $t$ relative to the final time of the simulations $t_{f}=1776$. As expected, $E$ agrees with the Sweet-Parker prediction $E_{\mathrm{SP}} \sim\left(\eta / L_{\mathrm{SP}}\right)^{1 / 2} \sim 0.011$, shown as the horizontal solid line. Here, $L_{\mathrm{SP}}$ is the half-length of the current sheet in the outflow direction; $L_{\mathrm{SP}} \sim L_{x} / 4$ since the full length takes up half the periodic domain. At $t=1200$, the Hall term is enabled. The lower dashed line shows $E$, revealing that reconnection remains slow.

A second series of simulations is initiated with the Hall effect enabled, but $\eta=0$. The reconnection is fast, as seen in the (blue) solid line in Fig. 6, where $t_{f}=802.15$. At $t=538.75$, a resistivity of $\eta=0.012$ is enabled. The upper dashed line shows $E$. While it does drop slightly (because the layer broadens slightly), it clearly remains much faster than $E_{\mathrm{SP}}$. In summary, the two dashed curves differ only in their time history, but are in different configurations, which demonstrate bistability even in the absence of electron inertia.

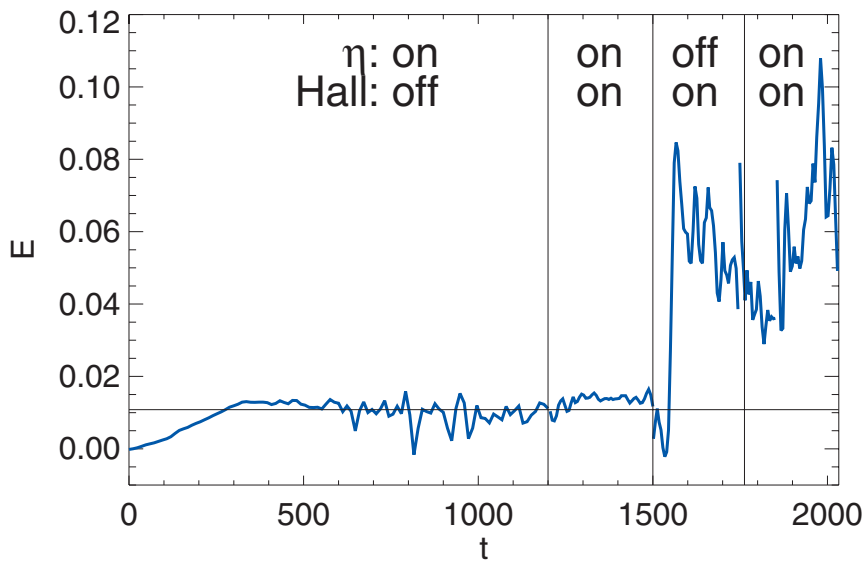

FIG. 8. (Color) Demonstration of hysteresislike behavior in the absence of electron inertia. Plotted is the reconnection rate $E$ as a function of time $t$. The system changes states when $\eta$ is changed from 0.012 to 0 and back. 
The out-of-plane current density $J_{z}$ is plotted in Fig. 7 with magnetic field lines superimposed for the Sweet-Parker and Hall reconnection solutions. The dramatic difference between the states is clearly seen. Note that the Sweet-Parker configuration exhibits a secondary island, which can induce a transition to fast reconnection. ${ }^{35,36}$ However, despite the island, fast reconnection does not occur.

While hysteretic behavior seems to follow directly from bistability, it is worth confirming with simulations (see Fig. 8 for $E$ versus $t$ ). The run is started in the slow reconnection regime using $\eta=0.012$. At $t=1500, \eta$ is zeroed and the reconnection becomes fast. The resistivity $\eta$ is then returned to its original value, and reconnection remains fast. Thus, removing and replacing $\eta$ leads to different physical states, which is hysteretic. Since the only nonresistive MHD effect is the Hall effect, it is the cause of bistability and hysteresis.

For completeness, we show the phase portrait for the evolution of the thickness $\delta$ for the simulation with zero electron mass as the dashed line in Fig. 4, which shows $d \delta / d t$ versus $\delta$. Even in the absence of electron inertia, one sees the bump in the plot which is a key signature of the saddle-node bifurcation. This behavior is further evidence that the Hall effect drives the dynamics.

\section{DISCUSSION}

In summary, we propose a model describing the nonlinear dynamics of magnetic reconnection using saddle-node bifurcations. This model is able to reproduce previously observed phenomena, such as hysteresis, bistability, and the existence of an unstable mode. ${ }^{3,10}$ We argue that the unstable mode is a balance between electron convection (ion convection plus the Hall term) and resistive diffusion. As a result, we provide a physical mechanism demonstrating that the dispersive behavior of the Hall effect causes instability of the mode and show why the dynamics of reconnection is described as a saddle-node bifurcation.

Results of two-fluid and Hall-MHD simulations are presented which are consistent with new predictions of this model. We show that the time evolution of the thickness of the layer follows the predicted behavior. We show that the outflow speed during a transition from Sweet-Parker to Hall reconnection is well described by the phase speed of the two-fluid wave. Finally, we show that the bistability and hysteresis continue to occur in a system without electron inertia, which implies that the Hall effect is the relevant physics for achieving bistability and hysteresis.

These results counter the claim ${ }^{23}$ that the cause of the hysteresis is electron inertia. It is outside the scope of this study to determine why previous analyses attain different results, but it should be addressed in future work. In particular, it has been emphasized ${ }^{20,21}$ that the models do not selfconsistently predict the length of the diffusion region, which makes them incomplete.

As mentioned in Sec. II, some previous authors studied Hall reconnection with $\eta$ balancing the reconnection electric field. ${ }^{15,17-19,22,23}$ The present results reveal that this state corresponds to the unstable solution, so this regime is physically unrealizable.
The present results do not address reconnection in pair plasmas. Such a system has no Hall effect, but has other additional physics due to the equal inertia of the two species. $^{16}$

The present study uses parameters where secondary islands do not play an important role (Lundquist numbers below $10^{4}$ ). However, for large Lundquist numbers, secondary islands qualitatively change Sweet-Parker reconnection. ${ }^{37}$ Their role remains under study. ${ }^{36,38-41}$ One result that is clear is that secondary islands lead to smaller length scales in the outflow direction, which causes the layers to become thinner in the inflow direction where they induce the onset of collisionless effects sooner than in the absence of secondary islands. ${ }^{35,36}$ However, collisionless reconnection still onsets catastrophically when the thinnest part of the Sweet-Parker layer reaches ion gyroscales. ${ }^{36}$ Thus, in making comparisons to physical systems, it is the current sheet thickness including secondary islands that should be used to compare to kinetic scales to see when transitions arise rather than the global Sweet-Parker scale lengths. ${ }^{36}$

\section{ACKNOWLEDGMENTS}

The authors have learned that a simulation study similar to that in Sec. III C is being carried out by B. P. Sullivan et al. (see Ref. 42).

The authors acknowledge valuable conversations with J. D. Huba. Computations used resources at the National Energy Research Scientific Computing Center. The authors acknowledge support by grants NSF Grant No. PHY-0902479 (P.A.C.), NSF Grant No. ATM-0645271 (M.A.S.), NASA Grant No. NNX08AM37G (M.A.S.), and NSF Grant No. PHY-0316197 (J.F.D.).

${ }^{1}$ X. Wang and A. Bhattacharjee, Phys. Rev. Lett. 70, 1627 (1993).

${ }^{2}$ Z. W. Ma and A. Bhattacharjee, Geophys. Res. Lett. 23, 1673, doi:10.1029/96GL01600 (1996).

${ }^{3}$ P. A. Cassak, M. A. Shay, and J. F. Drake, Phys. Rev. Lett. 95, 235002 (2005).

${ }^{4}$ P. A. Cassak, J. F. Drake, and M. A. Shay, Phys. Plasmas 14, 054502 (2007).

${ }^{5} \mathrm{P}$. A. Sweet, in Electromagnetic Phenomena in Cosmical Physics, edited by B. Lehnert (Cambridge University Press, New York, 1958), p. 123.

${ }^{6}$ E. N. Parker, J. Geophys. Res. 62, 509, doi:10.1029/JZ062i004p00509 (1957).

${ }^{7}$ M. E. Mandt, R. E. Denton, and J. F. Drake, Geophys. Res. Lett. 21, 73, doi:10.1029/93GL03382 (1994).

${ }^{8}$ M. A. Shay, J. F. Drake, B. N. Rogers, and R. E. Denton, Geophys. Res. Lett. 26, 2163, doi:10.1029/1999GL900481 (1999).

${ }^{9}$ J. Birn, J. F. Drake, M. A. Shay, B. N. Rogers, R. E. Denton, M. Hesse, M. Kuznetsova, Z. W. Ma, A. Bhattacharjee, A. Otto, and P. L. Pritchett, J. Geophys. Res. 106, 3715, doi:10.1029/1999JA900449 (2001).

${ }^{10}$ P. A. Cassak, J. F. Drake, M. A. Shay, and B. Eckhardt, Phys. Rev. Lett. 98, 215001 (2007).

${ }^{11}$ P. A. Cassak, J. F. Drake, and M. A. Shay, Astrophys. J. Lett. 644, L145 (2006).

${ }^{12}$ D. A. Uzdensky, Astrophys. J. Lett. 671, 2139 (2007).

${ }^{13}$ P. A. Cassak, D. J. Mullan, and M. A. Shay, Astrophys. J. Lett. 676, L69 (2008).

${ }^{14}$ A. N. Simakov, L. Chacón, and D. Knoll, Phys. Plasmas 13, 082103 (2006).

${ }^{15}$ L. Chacón, A. Simakov, and A. Zocco, Phys. Rev. Lett. 99, 235001 (2007).

${ }^{16}$ L. Chacón, A. Simakov, V. S. Lukin, and A. Zocco, Phys. Rev. Lett. 101, 025003 (2008)

${ }^{17}$ A. N. Simakov and L. Chacón, Phys. Rev. Lett. 101, 105003 (2008). 
${ }^{18}$ A. N. Simakov and L. Chacón, Phys. Plasmas 16, 055701 (2009).

${ }^{19}$ L. M. Malyshkin, Phys. Rev. Lett. 101, 225001 (2008).

${ }^{20}$ D. A. Uzdensky, Phys. Plasmas 16, 040702 (2009).

${ }^{21}$ B. P. Sullivan, A. Bhattacharjee, and Y.-M. Huang, Phys. Plasmas 16, 102111 (2009).

${ }^{22}$ L. M. Malyshkin, Phys. Rev. Lett. 103, 235004 (2009).

${ }^{23}$ A. Zocco, L. Chacón, and A. N. Sinakov, Phys. Plasmas 16, 110703 (2009).

${ }^{24}$ D. Tsiklauri, Phys. Plasmas 15, 112903 (2008).

${ }^{25}$ M. Hesse, S. Zenitani, M. Kuznetsova, and A. Klimas, Phys. Plasmas 16, 102106 (2009).

${ }^{26}$ S. H. Strogatz, Nonlinear Dynamics and Chaos (Addison-Wesley, Reading, 1994).

${ }^{27}$ B. N. Rogers, R. E. Denton, J. F. Drake, and M. A. Shay, Phys. Rev. Lett. 87, 195004 (2001).

${ }^{28}$ P. A. Cassak, Ph.D. thesis, University of Maryland, 2006.

${ }^{29}$ B. U. Ö. Sonnerup, in Solar System Plasma Physics, edited by L. J. Lanzerotti, C. F. Kennel, and E. N. Parker (North Halland, Amsterdam, 1979), Vol. 3, p. 46.

${ }^{30}$ M. Hesse, K. Schindler, J. Birn, and M. Kuznetsova, Phys. Plasmas 6, 1781 (1999)
${ }^{31}$ M. Hesse, M. Kuznetsova, and M. Hoshino, Geophys. Res. Lett. 29, 1563, doi:10.1029/2001GL014714 (2002).

${ }^{32}$ M. A. Shay and J. F. Drake, Geophys. Res. Lett. 25, 3759, doi:10.1029/ 1998GL900036 (1998).

${ }^{33}$ M. A. Shay, J. F. Drake, M. Swisdak, and B. N. Rogers, Phys. Plasmas 11, 2199 (2004)

${ }^{34}$ X. Wang, A. Bhattacharjee, and Z. W. Ma, J. Geophys. Res. 105, 27633, doi:10.1029/1999JA000357 (2000).

${ }^{35}$ K. Shibata and S. Tanuma, Earth, Planets Space 53, 473 (2001).

${ }^{36}$ W. Daughton, V. Roytershteyn, B. J. Albright, H. Karimabadi, L. Yin, and K. J. Bowers, Phys. Rev. Lett. 103, 065004 (2009).

${ }^{37}$ D. Biskamp, Phys. Fluids 29, 1520 (1986).

${ }^{38}$ N. F. Loureiro, A. A. Schekochihin, and S. C. Cowley, Phys. Plasmas 14, 100703 (2007)

${ }^{39}$ A. Bhattacharjee, Y.-M. Huang, H. Yang, and B. Rogers, Phys. Plasmas 16, 112102 (2009).

${ }^{40}$ P. A. Cassak, M. A. Shay, and J. F. Drake, Phys. Plasmas 16, 120702 (2009).

${ }^{41}$ P. A. Cassak and J. F. Drake, Astrophys. J. Lett. 707, L158 (2009).

${ }^{42}$ B. P. Sullivan, Y.-M. Huang, and A. Bhattacharjee, "On the question of hysteresis in Hall MHD reconnection," Phys. Plasmas (submitted). 